\title{
Pseudomembranous Erythematous Candida Stomatitis in an Immunocompetent Male Cameroonian: A Case Rapport
}

\author{
Nokam Abena Marie Elvire ${ }^{1, *}$, Bengondo Messanga Charles ${ }^{2}$, Chiabi Andreas ${ }^{3}$ \\ ${ }^{1}$ Stomatology Unit, The Cité Verte District Hospital, Yaoundé, Cameroon \\ ${ }^{2}$ Service Stomatology and Maxilla-facial Surgery Unit, The CHU, Yaoundé, Cameroon \\ ${ }^{3}$ Department of Pediatrics, Faculty of Medicine and Biomedical Sciences, University of Yaounde I, Cameroon \\ *Corresponding author: nokamabena@yahoo.fr
}

\begin{abstract}
Candida erythematous stomatitis is frequently seen in immunocompromised adults, and the first signs of immunodeficiency are seen in the oral cavity in which the weakened immune defense system, favors proliferation of saprophytic microbes. We report a case of candida pseudomembranous erythematous stomatitis in a 35year old immunocompetent Cameroonian male. Clinical examination showed erythematous lesions and white spots on the tongue, the internal face of the jaws, lower lips and the labial commissures. Mycologic tests showed the presence of fungi. The outcome was favorable with systemic and local anti mycotics and antiseptics.
\end{abstract}

Keywords: candida albicans, erythematous stomatitis, pseudomembranous candidiasis

Cite This Article: Nokam Abena Marie Elvire, Bengondo Messanga Charles, and Chiabi Andreas, "Pseudomembranous Erythematous Candida Stomatitis in an Immunocompetent Male Cameroonian: A Case Rapport." International Journal of Dental Sciences and Research, vol. 5, no. 3 (2017): 78-82. doi: 10.12691/ijdsr-5-3-5.

\section{Introduction}

Pseudomembranous and erythematous candida stomatitis also known as Oral candidiasis is an inflammatory lesion of oral mucosa and the tongue, resulting from a fungi infection known as Candida albicans [1].

Candida albicans is the main cause of oral candidiasis. A small quantity of fungi is normally found in the mouth but are usually neutralized by the immune system. A mycotic infection develops when the immune system is weakened and fungal invasion occurs [1,2].

Candida infections are opportunistic infections, the main predisposing factors are due to immunodepression which alters the normal flora of the mouth; that is why its affects more of the immune depressed patients whose immune system is fragile contrary to the immunocompetent [2,3].

The main favored factors are generals (HIV, diabetes, pregnancy, extreme age of live, dose antibiotics or corticoids) or locals (humidity, maceration and malocclusion) [2].

Candida infection is not only limited to the mouth; it can also be seen in different parts of the body. Clinical manifestations can be seen in different forms: Acute, subacute and chronic [4]. However, the clinical signs of oral candidiasis in all its forms (erythematous, pseudomembranous, angular cheilitis) must be diagnosed for rapid and effective treatment to alleviate functional problems as feeding, chewing, swallowing and speech [3,4,5].

Positive diagnosis takes into account the clinical aspect, localization, the general state and mycological examination $[2,4,5]$.
In 2003, the World Health Organization (WHO) published an article on oral lesions as indicators of human immunodeficiency virus and as predictive factors for the progression of HIV to Acquired immunodeficiency syndrome (AIDS) [1]. Oral manifestations are among the earliest and most important indicators of HIV infection $[1,4,5]$. Among oral lesions, oral candidiasis is the primary marker for HIV infection [1].

In our daily practice, in the presence of erythematous and pseudomembranous Candida stomatitis, HIV infection should be strongly suspected.

This case report describes and discusses the diagnostic aspects and therapeutic approach to patients with this disorder in order to improve the quality of life these patients in our context.

\section{Case Report}

Mr. NC, 35 years old driver, was referred of private clinic in February 2016 to the stomatology unit of the Cité verte district hospital in Yaounde, Cameroon, for the presence of oral lesions since three weeks. He had been diagnosed of having bacterial glossitis and placed on oral treatment of (Amoxicillin 1g + Clavulanic Acid 125mg) 2g daily and a Chlorhexidine mouthwash for 7 days with no improvement. He then consulted another dentist who prescribed (Spiramycin 1.5MUI + Metronidazole 250mg) 4 tablets daily for 10 days; this associated with symptomatic treatment: Anetholtrithione (Sulfarlem S 25*), for the mouth dryness; Paracetamol for pain relief. He had also been on oral gel consisting of association of 
Rhubarb and Salicylic Acid, and Chlorhexidine mouth wash. There was no improvement with this treatment.

Persistence of symptoms despite the above treatment motivated consultation in our hospital. The patient complained of oral sores, difficulty in eating, swallowing, talking and a dry mouth. He was not diabetic, does smoke nor drink alcohol, and had had chronic weight loss for several years and low back pain, which he treated with herbs made up of pawpaw and avocado leaves about 6 months ago.

He had general body weakness, asthenia, dysphagia and difficulty talking. Systemic examination was normal.

On examination we noted, synchrony of temporomandibular joints without audible sounds, and no palpable cervical adenopathy. There was presence of angular fissures and bilateral ulcerations of the labial commissures, with an inflammatory erythema towards the angular mucosae (Figure 1).

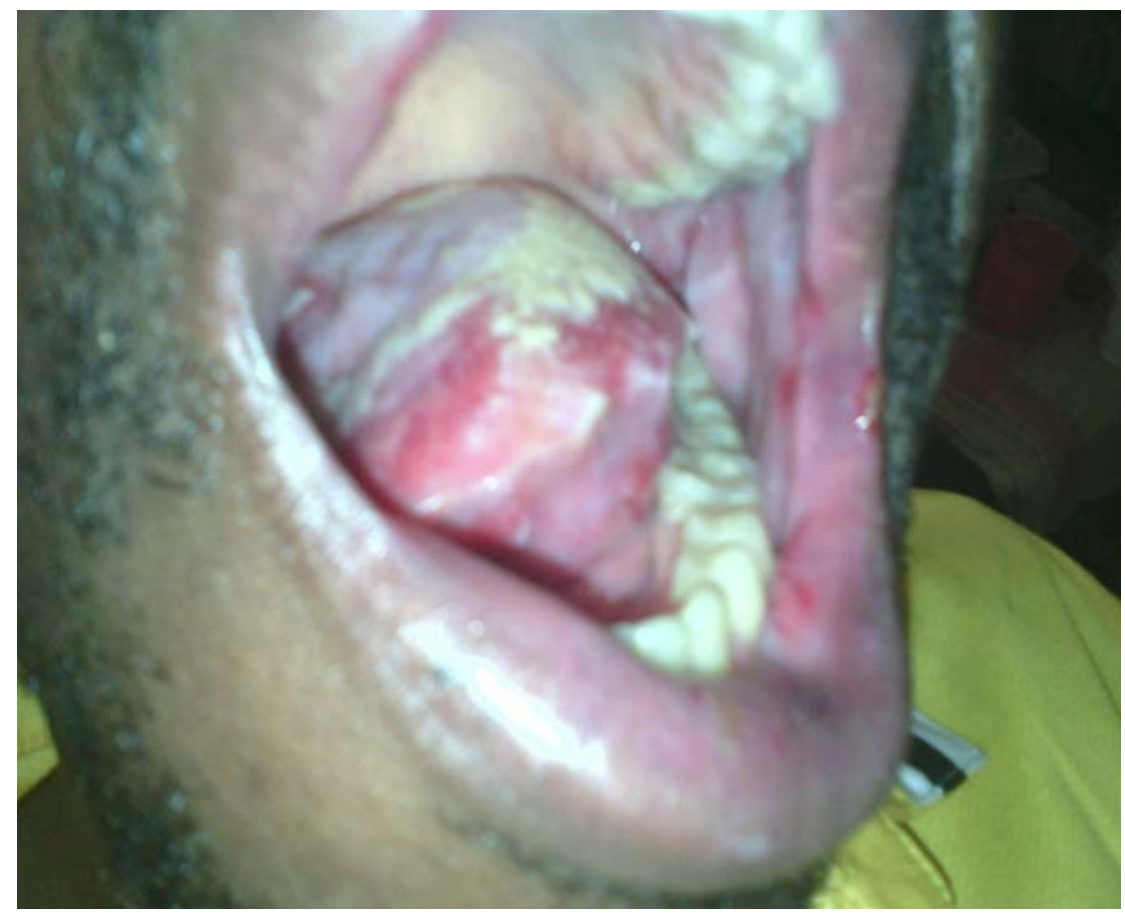

Figure 1. Erythematous and pseudomembranous stomatitis on the tongue, internal side of the jaw, and labial comer

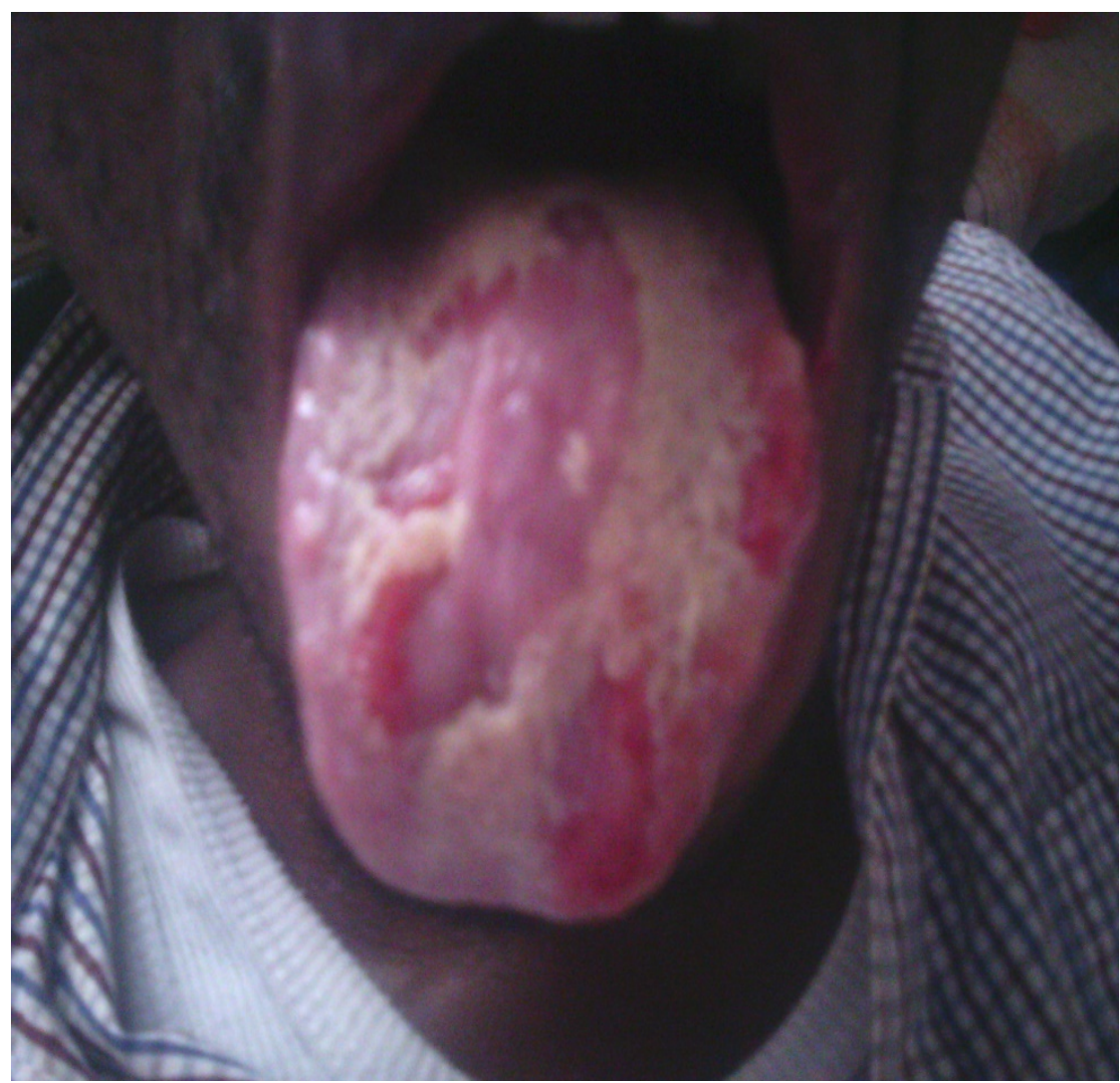

Figure 2. Geographical tongue with erythematous lesions with white plaques showing signs of oral candidiasis 


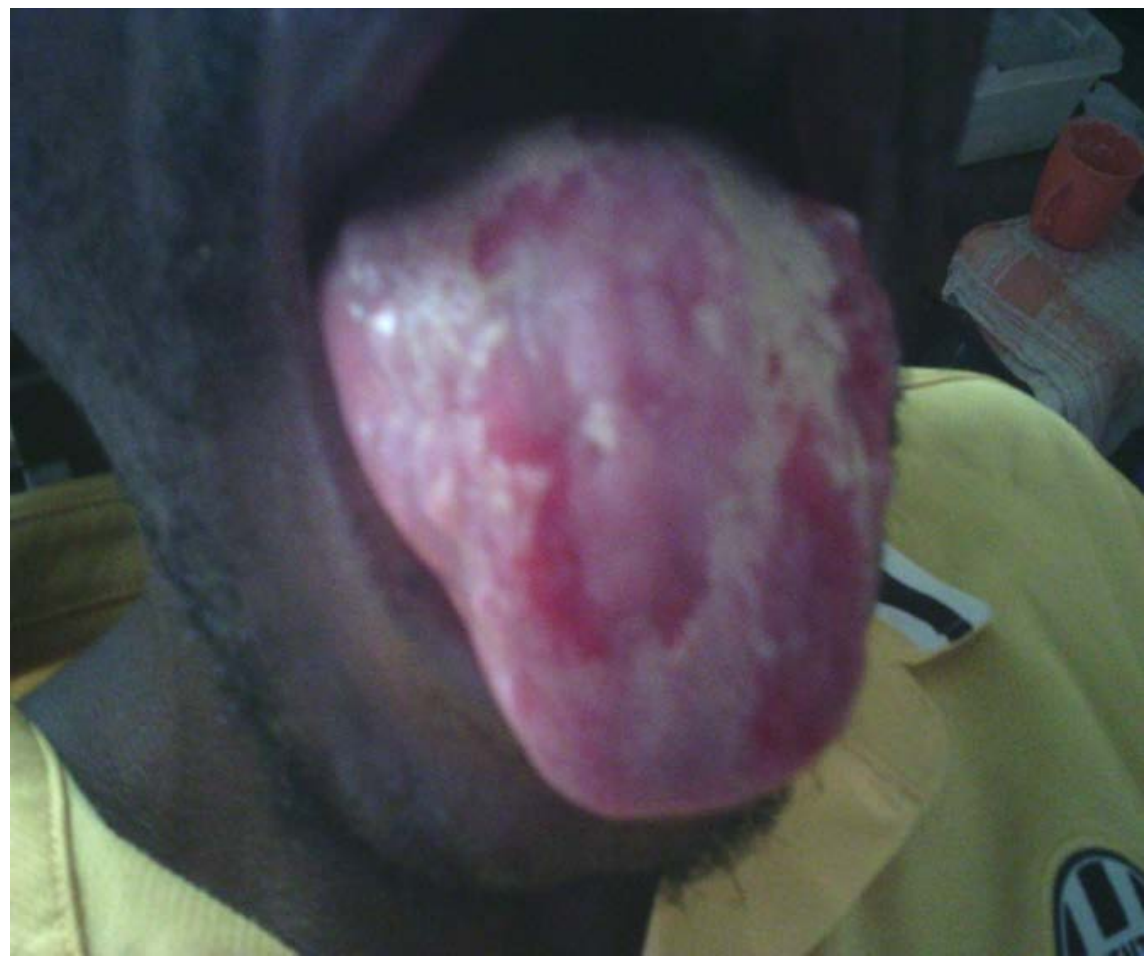

Figure 3. Oral candidiasis after two days

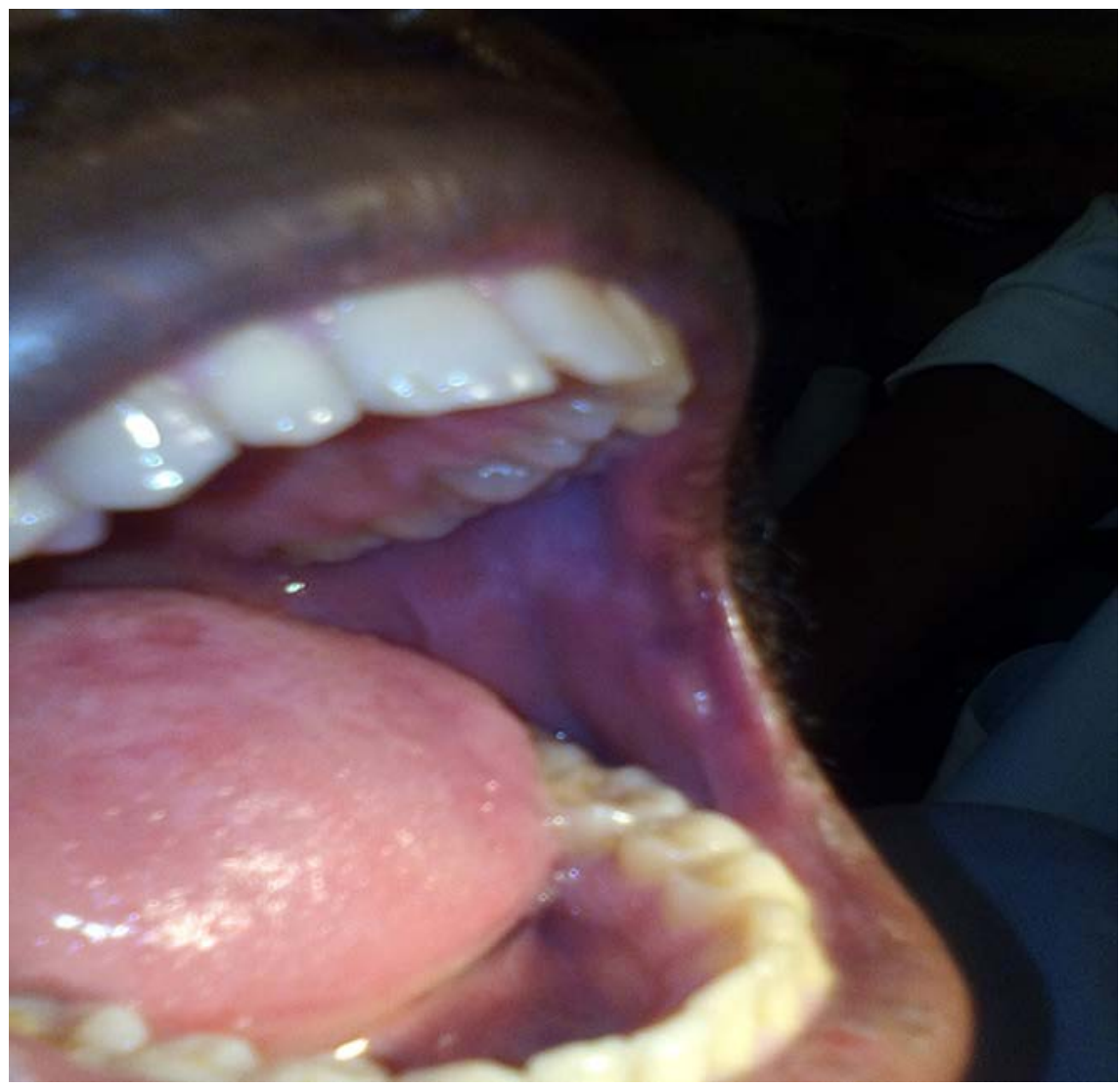

Figure 4. Disappearance of lesions on the buccal and angular commissure on the ninth day of treatment

In the mouth, there were multiple disseminated erythematous macular patches on the jugular mucosae, the lower labial commissure, and the vestibular gingiva facing the lower incisors and canines; depapillation of the dorsal part of the tongue, and bleeding when the whitish membrane was scrubbed off (Figure 1 and Figure 2). The patient had difficulty moving his mouth and tongue. Investigations done:
Complete blood count: Red blood cells: 5.12 x $10^{6} / \mu$; White blood cells: $4.47 \times 10^{3} / \mu$; Hematocrit: $42.1 \%$; Hemoglobin: $14.9 \mathrm{~g} / \mathrm{dl}$; Platelet count: $223 \times 10^{3} \mu \mathrm{l}$; HIV serology 1,2 and 0 by the Elfa / Vidas technique was negative. Fasting blood sugar was $0.9 \mathrm{~g} / \mathrm{l}$. Swab specimens of mucosal lesions for direct examination and culture for budding yeasts with presence of filaments were positive. 


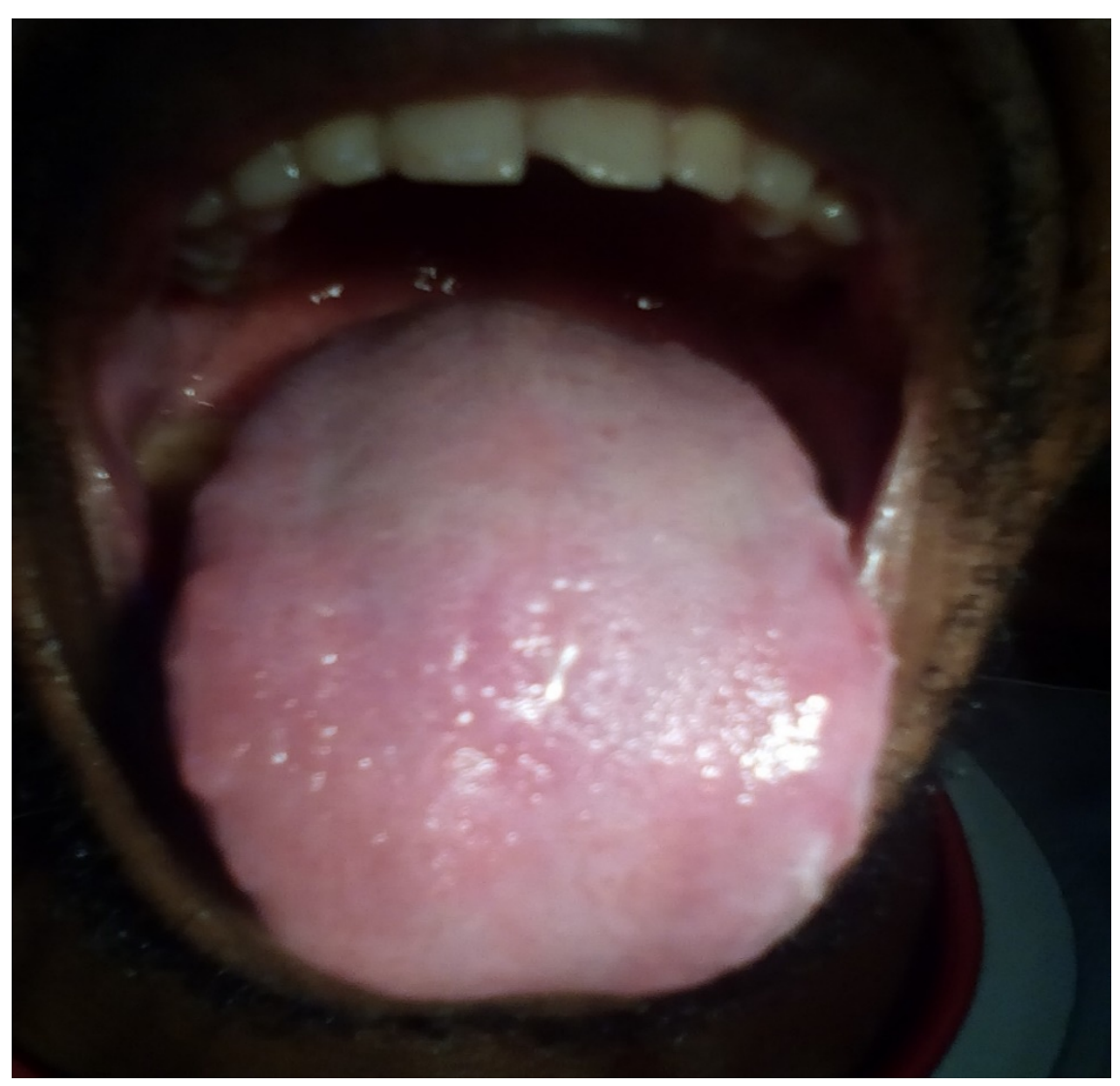

Figure 5. Complete disappearance of oral lesions on the twelfth day

Considering the morphology and topography of the lesions described above, we retained the diagnosis of erythematous and pseudomembranous candida stomatitis.

Initial treatment in the hospital consisted of an oral application of Xylocaine $10 \%$ topical gel, followed by oral hygiene using sterile gauze soaked in sodium bicarbonate and iodized Povidone solutions. The prescription consisted of Fluconazole 150mg one tablet daily for two weeks; Miconazol oral gel for local application 3 times daily, CaC1000 1 tablet daily; Sodium bicarbonate solution for mouthwash; Povidone iodine $10 \%$ solution for mouthwash; Clotrimazol solution for local application. This treatment is aim to stop the pain and proliferation of mycotic infection; this was permitted him to feed normally. He came for follow up two days after starting treatment (Figure 3). He had a good general status and was clinically improved. The burning sensations, dry mouth and whitish coatings had partially disappeared. The patient was seen successively on the fifth, and ninth days (Figure 4); and on the 12th day of treatment (Figure 5) the symptoms had completely disappeared. The mucous membrane became pink, and moist with normal salivary secretion.

\section{Discussion}

Several studies have emphasized the complexity of diagnosing oral lesions and consequently the difficulty in managing them. Glossitis is one of the disorders of the oral mucosa and has various causes; bacterial infection, vitamin deficiencies, allergic reactions, viral and mycotic attacks $[7,12]$.
The diagnosis in our patient was glossitis of bacterial origin in its initial phase. Initially, the patient would have had lesions on the tongue, which would have increased gradually over weeks to affect the mucous membranes, gums and the labial commissures. The functional signs were hypo salivation and painful oral sores, which led to difficulties in chewing, swallowing and speech, and could led to the anorexia and asthenia.

The diagnosis of erythematous and pseudomembranous Candida stomatitis is primarily clinical and on the topography of the lesions $[6,7,8]$. The positive diagnosis is based on functional and general signs and the type of lesions $[7,8]$.

The diagnosis of oral candidiasis in our patient was made from the intraoral lesions which showed an erythematous mucosa on the floor of the mouth (the dorsal aspect of the tongue, the gingiva, the internalsurfaces jaws, and labial commissures), which were coated with a whitish substance and which bled on scrubbing or cleansing of the tongue.

According to the World Health Organization (WHO), oral candidiasis is an opportunistic infection of the oral cavity in people living with HIV/AIDS. In Cameroon, a study carried out, showed that $18.4 \%$ of patients living with HIV/AIDS at the HIV/AIDS day care unit of Yaounde Central hospital had oral candidiasis [13].

The immune system eliminates invasive and pathogenic organisms, such as viruses, bacteria and fungi, while creating an equilibrium between the "good" and "bad" bacteria that normally reside in the body. Sometimes these protective mechanisms do not work. This may facilitate the development of oral candidiasis $[5,9,10]$.

Our patient was immunocompetent, with his HIV serology 1, 2 and 0 negative. 
An isolated angular cheilitis could have been caused by a bacterial infection or nutritional deficiency $[10,11]$. Our patient had Candida stomatitis associated with angular cheilitis. This could be explained by the licking tic that would have caused the spread of the infection on the angular commissure, thus creating the angular cheilitis. However, our therapeutic approach took into account probable vitamin deficiencies.

Diabetes may also contribute to the development of oral candidiasis. Uncontrolled diabetes, may increase the sugar level in the saliva, and this can favor growth of Candida albicans in the mouth $[7,10,12]$. He had no history of diabetes and his fasting blood sugar level was $0.9 \mathrm{~g} / \mathrm{l}$.

Oral manifestations caused by drugs are mainly candidiasis of different forms (thrush, geographical tongue and angular cheilitis). Broad-spectrum antibiotics make the body more vulnerable because they alter the natural flora of microorganisms; they destroy bacteria, which naturally prevents infections, thus promoting the multiplication of fungus as Candida $[6,7,8,10]$. The patient was on broad-spectrum antibiotics for about 20 days after his first medical visit. To treat the glossitis, he had taken Amoxicillin with clavulanic acid, and locally a mouthwash with Chlorhexidine for a week, followed by oral administration of Spiramycin and Metronidazole. This management would have favored proliferation of the Candida albicans in the mouth.

Oral candidiasis is a fungal infection whose various clinical forms are so often evident that diagnosis is evident from onset $[7,8,14]$.

Diagnosis of candidiasis is based on clinical examination. Confirmation by mycological tests is only necessary in atypical situations or in unusual localizations [7,8,9].

We did not see the necessity of taking a sample collection of the lesions for culture, because the clinical examination showed that it was an erythematous candida stomatitis. However, the positive response to local antifungal products (clotrimazol solution and oral miconazol gel) and oral administration (fluconazole tablets, 150mg daily for 14 days) confirmed diagnosis of oral candidiasis.

\section{Conclusion}

The aim of this case report was to describe and discuss the diagnostic aspects and therapeutic approach for erythematous and pseudomembranous candida stomatitis in an immunocompetent individual. Not all candida lesions are associated with HIV/AIDS. It has diverse clinical forms, depending on the general state of the patient and localization.

Clinically, diagnosis is based on the morphology and topography of the lesions and also on the functional signs, general body state, local factors and eventually mycologic tests.

The general plan of treatment take into consideration the general state of the patient, and local treatment of the oral cavity to ensure adequate feeding.

\section{References}

[1] Rapport ONUSIDA sur l'épidémie mondiale de SIDA 2010. http://www.unaids.org/globalreport/documents/20101123_Global Report_full_fr.pdf.

[2] Crickx, B., Géniaux, M., Bonerandi, J-J., Infections cutanéomuqueuses bactériennes et mycosiques - Infections cutanéomuqueuses à Candida albicans. Ann Dermatol Vénérol 2002; 129: 2S53-2S57.

[3] Samson, Ng., Prise en charge des patients atteints de candidose buccale. J Canadian Dental Association 2013; 79: d122.

[4] Melnick, S.L., Nowjack, R., Dushanka,V.R., Kleinman, P.A., Swango, Manifestations buccales de l'infection à VIH : Guide des études épidémiologiques. OMS Génève, 1994.

[5] Gasparin, A.B., Ferreira, F.V., Danesi, C.C., Mendoza-Sassi, R.A., Silveira, J., Martinez, A.M., Zhang, L., Cesar, J.A., Prevalence of oral lesions in persons with HIV and associated factors in a southern Brazilian city. Cad Saude Publica. 2009, 25(6): 1307-15.

[6] Chemlali, S., Kissa, J., Khlil, N., Chakib, A., Manifestations parodontales $d u \quad V I H$. Rapport ONUSIDA sur l'épidémie mondiale SIDA, 2012, 07: 19.

[7] Kulak,Y., Arikan, A., Kazazoglu, E., Existence of Candida albicans and microorganisms in denture stomatitis patients. J. Oral. Rehabil., 1997, 24: 788-790.

[8] Piette, E., Reychler, H., Lésions blanches de la muqueuse buccale et des lèvres. Encycl Med Chir. Paris : stomatologie, 22-045-k-10, 1997: 11.

[9] Parret, J., Bobillon, G., Lissac, M., Milieu buccal. Encycl Med Chir. Paris: 1982, 22008A10: 1-4.

[10] Baudet, P.M., Bertoin, P., Candidoses et prothèses. Actual. Odonto-Stomatol. 1993; 183: 431-437.

[11] Pindborg, J.J., Atlas des maladies de la muqueuse buccale. Paris: Masson, 1995:106-156j.

[12] Szpirglas, H., Ben Salma, L., Pathologie de la muqueuse buccale. Paris: Flammarion, 1997: 226-244.

[13] Ndjidda Bakari,W., Zoung-Kanyi Bissek, A.C., Tamoh, F.S., Tsague, T.N., Bengondo, M.C., Manifestations orales associées au VIH à l'Hôpital du jour de l'hôpital centrale de Yaoundé, la communication orale, $5^{\text {eme }}$ Congrès dentaire international ANCDS, Dakar 25.

[14] Samson, J., Candidoses buccales: épidémiologie, diagnostic et traitement. Rev. Mens. Suisse Odonto-stomatol, 1990, 100; 5: 548-557. 